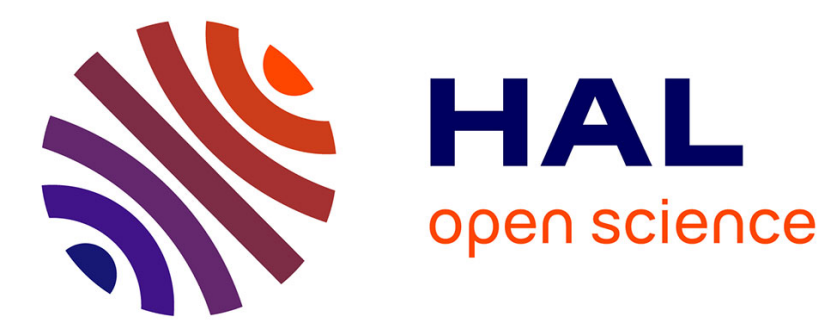

\title{
A framework to Calibrate a MEMS Sensor Network
} Kahina Boutoustous, Eugen Dedu, Julien Bourgeois

\section{To cite this version:}

Kahina Boutoustous, Eugen Dedu, Julien Bourgeois. A framework to Calibrate a MEMS Sensor Network. UIC'09, 6-th Int. Conf. on Ubiquitous Intelligence and Computing, 2009, Australia. pp.136149. hal-00661804

\section{HAL Id: hal-00661804 https://hal.science/hal-00661804}

Submitted on 20 Jan 2012

HAL is a multi-disciplinary open access archive for the deposit and dissemination of scientific research documents, whether they are published or not. The documents may come from teaching and research institutions in France or abroad, or from public or private research centers.
L'archive ouverte pluridisciplinaire HAL, est destinée au dépôt et à la diffusion de documents scientifiques de niveau recherche, publiés ou non, émanant des établissements d'enseignement et de recherche français ou étrangers, des laboratoires publics ou privés. 


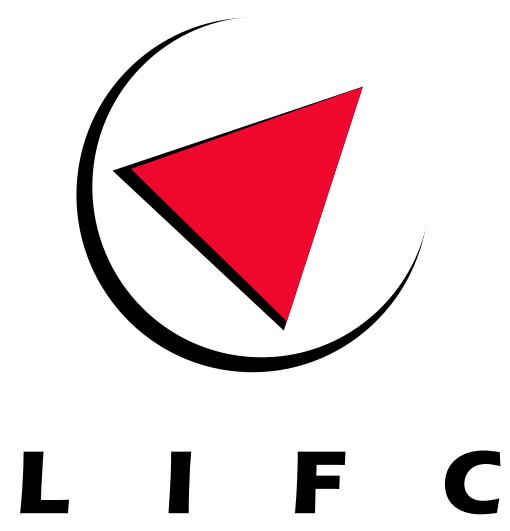

LABORATOIRE D'INFORMATIQUE DE L'UNIVERSITE DE FRANCHE-COMTE

EA 4269

\section{A framework to Calibrate a MEMS Sensor Network}

Kahina Boutoustous — Eugen Dedu — Julien Bourgeois

\section{Rapport de Recherche no RR 2011-????}

THÈME 4 - Juillet 2009 



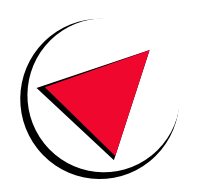

L I $\mathbf{F}$ C

\title{
A framework to Calibrate a MEMS Sensor Network
}

\author{
Kahina Boutoustous , Eugen Dedu , Julien Bourgeois
}

Thème 4

OMNI

Juillet 2009

\begin{abstract}
The Smart Surface project aims at designing an integrated micro-manipulator based on an array of micromodules connected with a 2D array topology network. Each micromodule comprises a sensor, an actuator and a processing unit. One of the aims of the processing unit is to differentiate the shape of the part that is put on top of the Smart Surface. From a set of shapes this differentiation is done through a distributed algorithm that we call a criterion. The article presents Sensor Network Calibrator (SNC), a calibrator which allows to parametrize the Smart Surface and to determine the necessary number of sensors required by our Smart Surface. The tests will show that SNC is of great importance for choosing the number of sensors, and therefore to determine the size of the sensors grid.
\end{abstract}

Key-words: shape differentiation, distributed computing, MEMS 



\section{Une plate-forme pour calibrer un réseau de capteurs MEMS}

Résumé :

Mots-clés :

Laboratoire d'Informatique de l'Université de Franche-Comté, Antenne de Montbéliard — UFR STGI,

Pôle universitaire du Pays de Montbéliard, 25200 Montbéliard Cedex (France) 



\section{Introduction}

During an assembly process, it is necessary to feed assembly line workstations with well-oriented and well-positioned parts. These parts are often jumbled and they need to be sorted and conveyed to the right workstation. To do so, the operations to be performed on parts are the following: identifying, sorting, orienting, positioning, feeding, and assembling. Among the most promising solutions to perform these tasks on microparts, is the combination of micro-electro mechanical systems (MEMS) in order to form an actuator arrays. However, if a single microactuator is not powerful enough to move a micropart, several microactuators working cooperatively might very well do it. A MEMS sensor/actuator array with embedded intelligence is referred as a Smart Surface.

The objective of the Smart Surface project is to design such an integrated MEMS system which will be able to identify, to sort, to orient and position microparts. This article deals only with the identification part of the process: A micropart is put on the Smart Surface which have to recognize the part shape among several models and give the proper orders to the control system to move it on the right place. In fact, recognition is not the proper term. Given a set of parts, the Smart Surface will have to differentiate all the parts within the set. As the processing power of the Smart Surface is embedded in very limited space, this differentiation process has to be optimized both in term of memory used and processing power needed. The differentiation is made by a distributed program loaded in the Smart Surface.

The aim of the Sensor Network Calibrator SNC which is presented in this article is to parametrize our Smart Surface, i.e. to find the size of the sensors grid necessary to differentiate our models. For that, a Smart Surface platform consisting of a Smart Surface prototype with a camera positioned above it is used. The rest of the paper is organized as follows. Section 2 details the Smart Surface project. Section 3 presents the SNC, while the tests are performed on section 4 . Some related works to shape representation are presented in section 5 and they are followed by conclusions and presentation of future works.

\section{The Smart Surface Project}

There have been numerous projects of MEMS actuator arrays in the past and more precisely in the 1990's. These pioneer researches have developed different types of MEMS actuator arrays, based on actuators either pneumatic [1,2], servoed roller wheels [3,4], magnetic [5] or thermobimoph and electrostatic [6]. Some of these preliminary studies use a sensorless manipulation scheme based on Goldberg's algorithm [7] for parallel jaw grippers. The jaw grippers are obtained with MEMS actuator arrays by creating opposite field forces which then can orient and move the parts. Bohringer et al. [8] have proposed a concept called "programmable force field" which is an extension of Goldberg's algorithm. This manipulation scheme which is well-adapted for jaw grippers has shown some limitations when adapted to MEMS actuator arrays. For instance, the absence 
of a command law can lead to uncertain behaviours [9] or MEMS actuator arrays have to be programmed for each different kind of parts. More recent research has been conducted in order to include sensors and to add intelligence to MEMS actuator arrays but it either fails to develop it at a micro-scale [10] or to be fully integrated [11].

The objective of the Smart Surface project is to design a distributed and integrated micro-manipulator based on an array of micro-modules in order to realize an automated positioning and conveying surface. Each micro-module will be composed of a micro-actuator, a micro-sensor and a processing unit. The cooperation of these micro-modules thanks to an integrated network will allow to recognize the parts and to control micro-actuators in order to move and position accurately the parts on the Smart Surface. The parts are small, they cover a few numbers of micro-modules (e.g. $4 \times 4$ ).

Figure 1 shows the Smart Surface. The rectangular holes seen on the frontside are the air nozzles. Air-flow comes through a micro-valve in the back-side of the device and then passes through the nozzle. The advantage of this solution is that the micro-actuators, the most fragile part of the surface, are protected. The circle holes are used by the micro-sensors to detect the presence or not of the part on the surface.

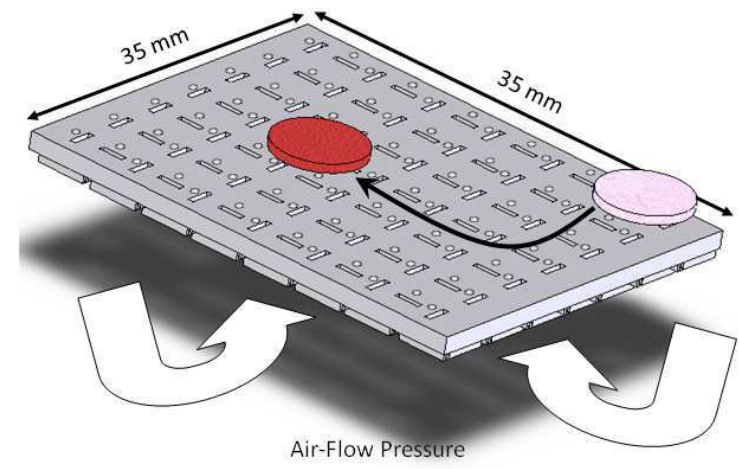

Figure 1. An overview of our Smart Surface.

The strength of our project is the multidisciplinary collaboration between six labs specialized in their field and more than twenty researchers. We are responsible for the information management inside the Smart Surface, i.e. distributed differentiation of the part and communication infrastructure. 


\section{The SNC Calibrator}

One of the parameters of the Smart Surface is its number of sensors. Due to space restriction, this number has to be chosen as wisely as possible: if it is overestimated, the design of the SS will be impossible, if it is underestimated, the part differentiation will also be impossible. It is therefore a crucial parameter that has to be set.

\subsection{Experimental platform}

The Smart Surface is still in design phase and it is noticeably easier to construct a Smart Surface Prototype (SSP) at a greater scale than the micro-scale SS. So, until its final fabrication, a Smart Surface platform has been built, which consists of Smart Surface Prototype (SSP), with a camera positioned above it (see fig. 2).

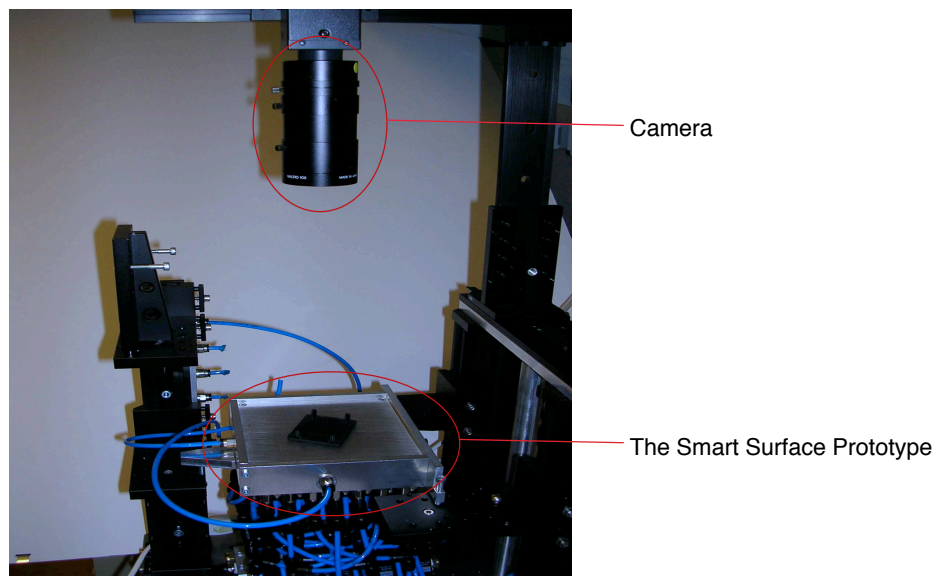

Figure 2. Overview of the Smart Surface platform.

The Smart Surface Prototype (SSP) is a $30 \times 30 \mathrm{~cm}$ square surface with a $15 \times 15$ actuators array. The air flow wich comes through actuators continuously moves the part on SSP. A camera positioned above the SSP allows free discretization of the part.

The Smart Surface platform allows us to perform real experiments.

\subsection{SNC overview}

This section presents the calibrator that has been implemented to define the number of the sensors to be embedded into the Smart Surface. The calibrator presented in fig. 3 receives as input: 
- the video from the camera which is positioned above the SSP; this camera shoots the SSP, as well as the parts on top of it;

- the group of models which is recognized by the SSP. A model is a part which is passed as a parameter;

- the sizes of the sensors grid to test.

The calibrator provides as output the result of the differentiation that is an answer to the following question: For each given size of sensors grid, what is the differentiation rate?

For each model, a set of characteristics is defined: area, perimeter, etc. We will refer to them as criteria and their combination will be called the combination of criteria [12]. When a part is on the Smart Surface, the aim is to differentiate it among the various models given to the Smart Surface.

The differentiation consists of calculating the values of the criteria of the part on the Smart Surface, then these values will be compared with the values of the criteria of the models. The result of this comparison is the differentiation rate [12].

In our previous paper [12] we presented as future work the free rotation. In the current paper we present this free rotation. Another aspect of this paper is that it does not do exhaustive research, but only a few various models.

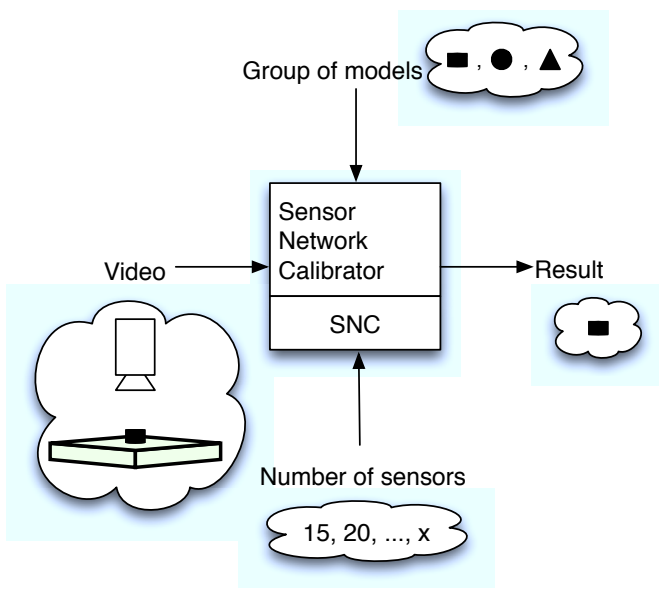

Figure 3. Overview of the calibrator.

Fig. 4 presents the structure of our calibrator. The calibrator has two phases:

1. Offline: For each group of models of the SSP and each size of sensors grid, find the best combination of criteria (and their masks, see later) for the differentiation of the models between each other [12]. 


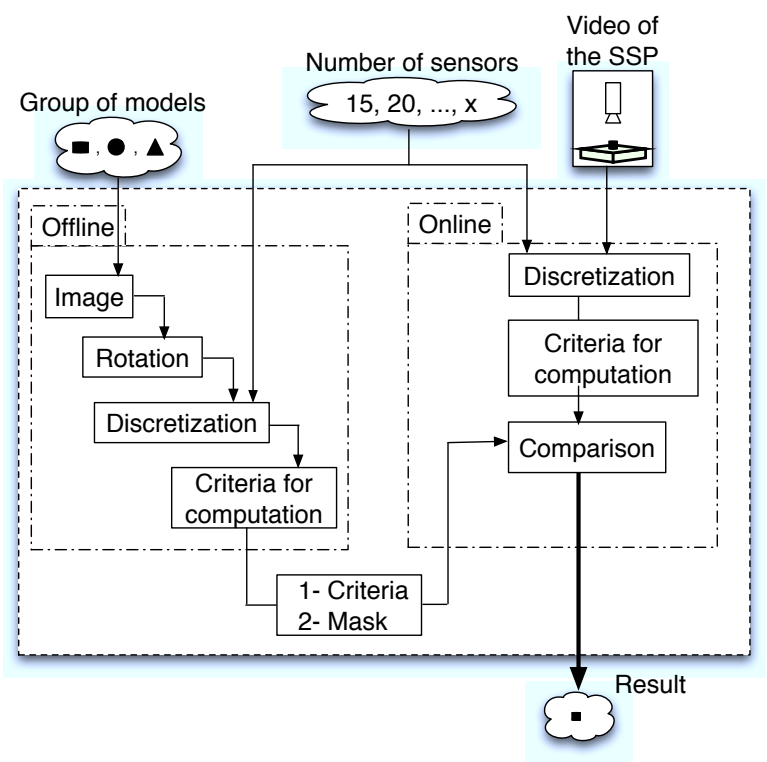

Figure 4. Global structure of our calibrator.

2. Online: An attempt to differentiate the part on the SSP is made using a differentiation algorithm, the various size sensors grid given as input and the results of the first offline phase.

\subsection{Offline phase}

In this phase, for each group of models, to be differentiated among them, all combinations of criteria are determined. This phase is subdivided into five subphases:

1. all rotations ${ }^{1}$ of $1^{\circ}$ are generated, $M R_{R} t_{i}$, from the image of the model $M_{i}$, given as input, with respect to the centre of the image, and all translations of width(MRot)/10 pixels are generated from the image $M R_{\text {Rot }}$;

2. a grid corresponding to the positions of the sensors (middle point of the cell) is drawn on the images;

3. the images are discretized in a matrix by affecting 1 if the sensor is covered by the part and 0 otherwise; in order to have negligible errors for the rotation, the resolution of the image should be much greater than the resolution of the SSP;

4. unique masks corresponding to the initial matrix without the rows and columns that contain only zeros are saved;

\footnotetext{
${ }^{1}$ The OpenCV library was used for rotations and translations.
} 
5. the values of each criterion are calculated for all masks of the model.

The following algorithm details these 5 sub-phases:

1: for each size of sensors grid $(n, n)$, with

$n \subset 15,20,25,30, \ldots, 50$ do

2: $\quad$ for each $M_{i} \subset M_{1}, M_{2}, M_{3}, \ldots, M_{n b r_{-} \text {models }}$ do

3: $\quad$ Acquire the image $I m$ of the model on the SSP

4: $\quad$ for $d=1^{\circ}$ to $360^{\circ}$ do

5: $\quad$ Generate $\operatorname{ImRot}$ by rotating the image $I m$ by $d$ degrees

6: $\quad$ for each $y \subset 0,10,20,30, \ldots, \operatorname{Size}($ ImRot $)$ do

7: $\quad$ for each $x \subset 0,10,20,30, \ldots$, Size (ImRot) do

8: $\quad$ Generate ImTrans by translating the image ImRot by $x$ steps on $O x$ and $y$ steps on $O y$

9: $\quad$ Discretize the image ImTrans

10: $\quad$ Generate and save the mask

11: $\quad$ Calculate and save the value for each criterion

12: $\quad$ end for

13: $\quad$ end for

14: $\quad$ end for

15: end for

16: end for

Among all the calculated criteria, the best criterion is chosen according to the differentiation rate, the memory cost and the execution time [12]. Finally, this criterion and its masks are given as input to the online phase.

\subsection{Online phase}

This phase consists of differentiating the part on the SSP, which means determining to which model recognized by the SSP it is associated. This phase is divided into four sub-phases:

1. the camera positioned above the SSP takes a sequence of images of the SSP and the part on it;

2. each image obtained is discretized (the image has only black and white pixels) to each grid size given as input to SNC, and the mask of the binary representation of this part is extracted;

3. the values of all combinations of the criteria of the masks are calculated;

4. the values of the criteria are compared with the values of the criteria of the models, to differentiate the part on the SSP.

The algorithm below describes this phase:

1: Film the part on the SSP

2: Generate the result tree of criteria values of the models recognized by the SSP

3: for each image of the part on the SSP do

4: $\quad$ Discretize the image

LIFC 


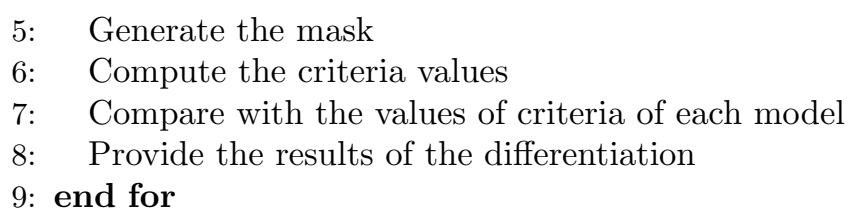

\section{Tests}

The aim of our work is to parametrize the Smart Surface, i.e. finding the right size of the sensors grid to be used for differentiating [12] groups of given models (fig. 5 shows one of these models on the SSP). For this, several tests have been performed on the SSP.

The differentiation is made by computing the different criteria and by applying a differentiation algorithm [12].

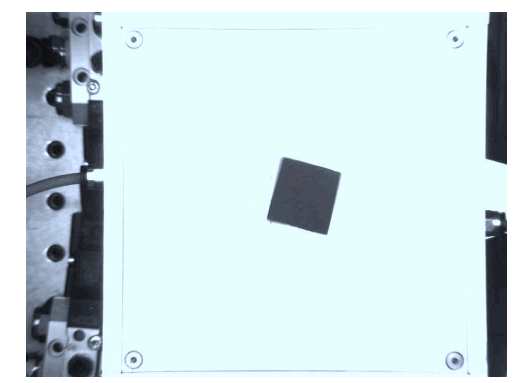

Figure 5. Picture of the SSP with a square on it.

A set of four basic models have been chosen (see fig. 6(a)). Starting from these models all group of three models have been generated (see fig. 6(b)-(e)). Several sizes of grid sensors are used see below.

\subsection{Offline phase}

The offline algorithm is applied to each group of models as already described, using images of size 550x 550 , a rotation step of $1^{\circ}$, a translation step of 10 pixels and different grid sizes of the sensors $((15,15)(20,20), \ldots,(50,50))$.

The offline phase consists of computing the values for differentiation criteria [12] calculated for the models $M_{1}, M_{2}, M_{3}$. Fig. $7($ a) represents an example of results of the offline phase, for the models $L, I, O$, the last group in fig. 6(e) with $D, C, A$ differentiation criteria :

- D: The sum of 1 located on both diagonals.

- C: The sum of the number of $\mathrm{V}$ shape angles. 


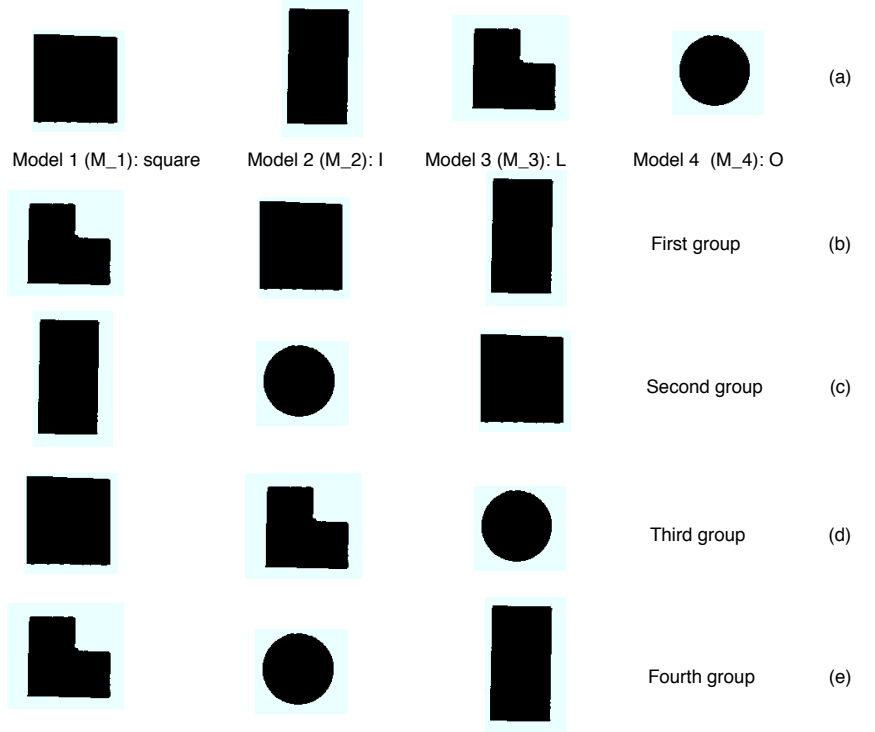

Figure 6. (a) Definition of our models, (b)-(e) all groups of three models.

- A: The number of 1 having at least three neighbors to 0 and forming a right angle.

Figure 7(b) shows a generated tree according to the results of figure 7(a). This tree is just a representation of results for the obtained criteria.

\subsection{Online phase}

The aim of this phase is to determine for each group of models, the size of a grid of sensors that allows differentiating our models. For this, the differentiation rate for each part on the SS with each model must be calculated

The algorithm of the online phase is applied to the group of models $\{L, I, O\}$ (see fig. 6(e)). The results are shown in fig. 8, representing the differentiation rate for the $L, I$ and $O$ belonging to group $\{L, I, O\}$.

The size of the sensors grid of the SSP must be determined such that it allows a better differentiation rate for all groups of models. For that, the average ${ }^{2}$ differentiation rate of our models has been computed. Fig. 8 shows also the average differentiation rate of group $\{L, I, O\}$. It can be easily observed that with 35 sensors a higher differentiation rate average is obtained. With the same number of sensors (35) the following results are obtained:

\footnotetext{
${ }^{2}$ We consider that the models have the same probability (33\%) to be put on the SSP, otherwise weights should be used to compute the average.
} 

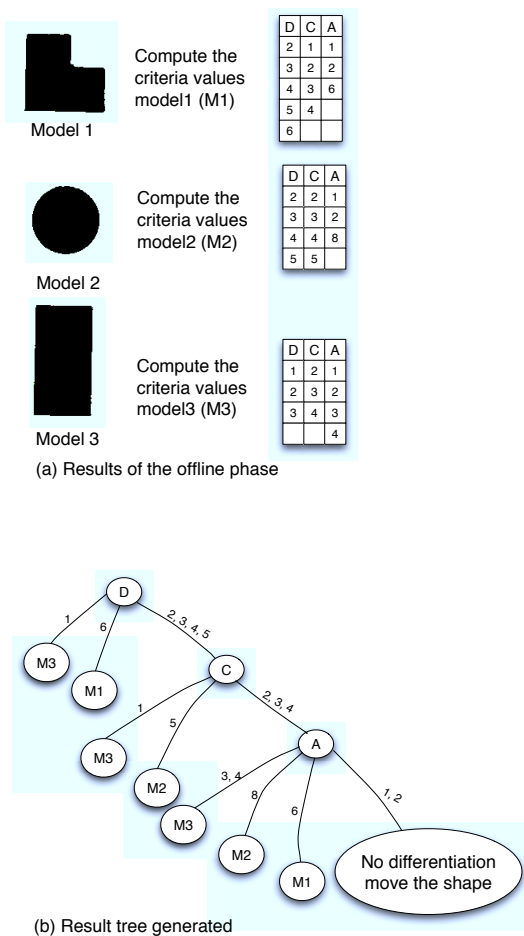

Figure 7. An example of the result tree of criteria values according to a combination of criteria and a set of models.

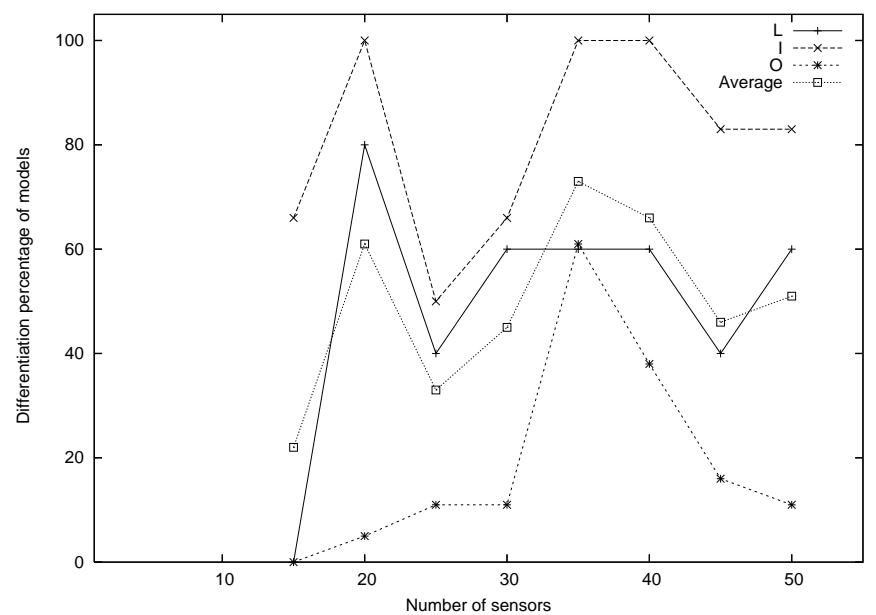

Figure 8. Differentiation percentage of models $L, I, O$, and their average. 
- For the $L$, the differentiation rate is $60 \%$. It's not the highest value but it's a satisfactory result.

- For the $I$, the highest differentiation rate is $100 \%$.

- For the $O$, the highest differentiation rate is $61.11 \%$.

Fig. 9, fig. 10 and fig. 11 contain the differentiation rate of each model belonging to each group of models: $\{$ square $, I, L\},\{$ square $, L, O\}$ and $\{$ square $, I, O\}$ respectively (see fig. 6(b), fig. 6(c), fig. 6(d)) as well as the average differentiation rate for each of them.

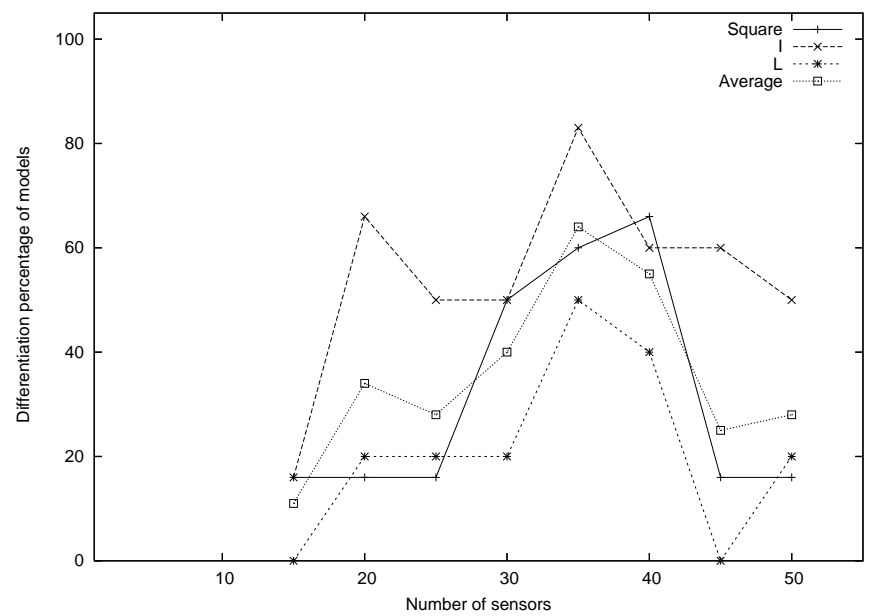

Figure 9. Differentiation percentage of models square, $I, L$, and their average.

Taking into account the values from fig. 9, fig. 10 and fig. 11 we can state that a sensors grid of $(35,35)$ is an appropriate parameter for the SSP because a high differentiation rate average is obtained.

Logically, we thought that the differentiation rate would increase proportionally to the number of sensors but the results have shown that, starting from a threshold (35 sensors) the differentiation rate decreases. This is caused by the increasing number of information to manage which implies an increase of the binary representations.

\section{Related work}

Several methods of shape representation exist in the literature. They are divided into two categories: contour-based methods and region-based methods. Contourbased methods are widely used. But generally, for complex images, the contour is not enough to describe the image content, therefore region-based methods are better. 


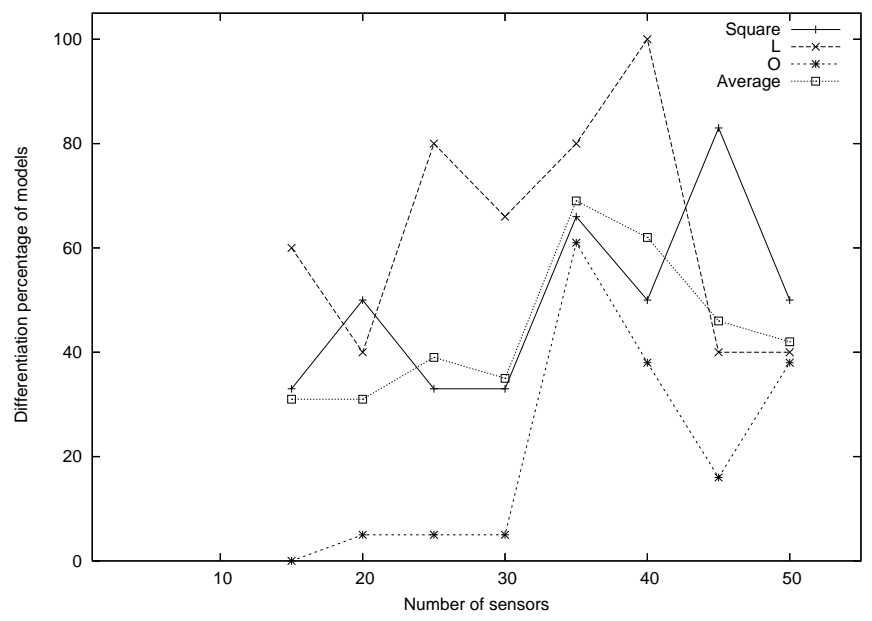

Figure 10. Differentiation percentage of models square, $L, O$, and their average.

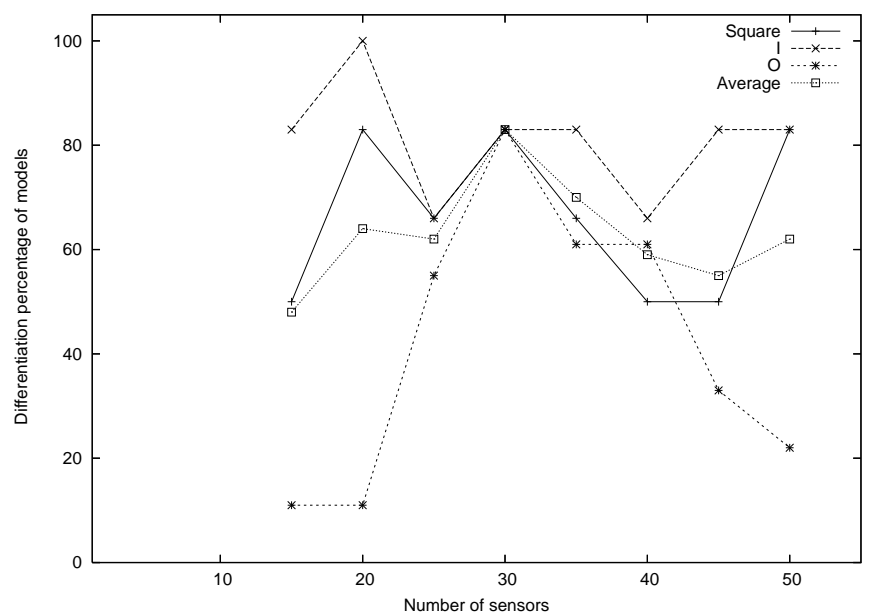

Figure 11. Differentiation percentage of models square, $I, O$, and their average. 


\subsection{Contour-based approaches}

In the contour-based approach, the pixels of the contour are considered.

Fourier descriptors This approach is divided into two steps:

1. The image is defined by a one-dimensional function called shape signature, which is nothing else than a compact representation of the image [13]. Many methods to calculate the signature have been developed. The most common shape signatures are: centroid distance [14], [15], chordLength signature [16] and area function.

2. Once the shape signature has been calculated, a Fourier transform is applied [17], [18]. It results in coefficients called Fourier descriptors of the shape. These descriptors represent the shape of the object in the frequency domain. The Fourier transform is invariant against translation, scale, rotation and their starting point.

Freeman code Freeman coding consists in browsing the borders of the shape with elementary moves from a starting point and coding the movement [19], [20].

Freeman code is sensitive to rotation because Freeman code depends on the starting point. To reduce this dependence, the resulting number has to be the minimal. The Freeman code is invariant to translation. It is also invariant to a rotation of $90^{\circ}$ for the 4 -connectivity and $45^{\circ}$ for the 8 -connectivity [21], [22].

Fourier descriptors and Freeman code are widely used for big pictures where the outline of the image differs noticeably from the inside of the images (parts). In our study these methods are not very interesting given that we are working on tiny images where the contour is equal or nearly equal to the surface.

\section{$5.2 \quad$ Region-based approaches}

In region-based methods, all the pixels within a shape are taken into account to obtain the shape representation.

Grid based In this method [23], a fixed-length grid of cells on the image is drawn. Going along our grid from top to bottom and from left to right, each cell that is wholly or partly covered by the form is affected with the value 1 , and other cells with 0 [24]. This produces a binary number, which is the representation of our shape. The difference between two parts is given by an XOR between their binary representations. Such a binary representation is very sensitive to rotation, translation and dilatation, that is it requires a prestandardization. 
Invariant moments In this method [25], [26], [27] the invariant moments are used to represent the image. A set of seven descriptors exist, called Husont invariants, computed by normalizing central moments of order three. They are invariant to object scale, translation and orientation. They are used as input vectors for the classification method. There are several classification methods, among them neural networks are the most widely used because of their fault tolerance, their ability of classification and their generalizability. The invariant moments are widely used in three dimension models or large images that need to be compacted. It is not very useful to apply this method in our case because the images are very small.

\section{Conclusions and future works}

In this article we present the SNC calibrator which allows to parametrize our Smart Surface by determining the required number of sensors. Our tests performed on all groups of 3 out of 4 models show that a sensors grid of $(35,35)$ is an appropriate parameter for the Smart Surface.

One of the ideas of our future work is to test more models with the SNC calibrator, and with a real Smart Surface when this will be operational. Also, to develop distributed algorithms for various criteria in order to implement them in the Smart Surface.

\section{References}

1. Pister, K., Fearing, R., Howe, R.: A planar air levitated electrostatic actuator system. In: IEEE Workshop on Micro Electro Mechanical Systems. (1990) 61-71

2. Fujita, H.: Group work of microactuators. In: International Advanced Robot Program Workshop on Micromachine Technologies and Systems, Tokyo, Japan (October 1993) 24-31

3. Luntz, J.E., Messner, W.: A distributed control system for flexible materials handling. IEEE Control Systems 17(1) (February 1997)

4. Luntz, J.E., Messner, W., Choset, H.: Parcel manipulation and dynamics with a distributed actuator array: The virtual vehicle. In: IEEE Int. Conf. on Robotics and Automation (ICRA). (1997) 1541-1546

5. Liu, C., Tsao, T., Will, P., Tai, Y., Liu, W.: A micromachined permalloy magnetic actuator array for micro robotics assembly systems. In: The 8th International Conference on Solid-State Sensors and Actuators. (1995)

6. Suh, J., Glander, S., Darling, R., Storment, C., Kovacs, G.: Combined organic thermal and electrostatic omnidirectional ciliary microactuator array for object positioning and inspection. In: Solid State Sensor and Actuator Workshop. (1996)

7. Goldberg, K.Y.: Orienting polygonal parts without sensors. Algorithmica 10(2-4) (1993) 210-225

8. Böhringer, K.F., Bhatt, V., Donald, B.R., Goldberg, K.Y.: Algorithms for sensorless manipulation using a vibrating surface. Algorithmica 26(3-4) (2000) 389-429

9. Murilo, G.C., Peter, M.W.: A general theory for positioning and orienting $2 \mathrm{~d}$ polygonal or curved parts using intelligent motion surfaces. In: ICRA. (1998) $856-862$ 
10. Biegelsen, D., Berlin, A., Cheung, P., Fromherts, M., Goldberg, D., Jackson, W., Preas, B., Reich, J., Swartz, L.: Airjet paper mover. In: SPIE Int. Symposium on Micromachining and Microfabrication. (2000)

11. Fukuta, Y., Chapuis, Y.A., Mita, Y., Fujita, H.: Design, fabrication and control of MEMS-based actuator arrays for air-flow distributed micromanipulation. IEEE Journal of Micro-Electro-Mechanical Systems 15(4) (August 2006) 912-926

12. Boutoustous, K., Dedu, E., Bourgeois, J.: An exhaustive comparison framework for distributed shape differentiation in a MEMS sensor actuator array. In: International Symposium on Parallel and Distributed Computing (ISPDC), Krakow, Poland, IEEE computer society press (July 2008) 429-433

13. Loncaric, S.: A survey of shape analysis techniques. Pattern Recognition 31(8) (1998) 983-1001

14. Zhang, D., Lu, G.: Content-based shape retrieval using different shape descriptors: A comparative study. In: ICME, IEEE Computer Society (2001)

15. Zhang, D., Lu, G.: Shape-based image retrieval using generic Fourier descriptor. SPIC 17(10) (November 2002) 825-848

16. Zhang, D., Lu, G.: Study and evaluation of different Fourier methods for image retrieval. Image Vision Comput. 23(1) (2005) 33-49

17. Diaz de Léon, R., Sucar, L.: Human silhouette recognition with Fourier descriptors. In: ICPR00, vol III. (2000) 709-712

18. Derrode, S., Daoudi, M., Ghorbel, F.: Invariant content-based image retrieval using a complete set of Fourier-Mellin descriptors. In: ICMCS, Vol. 2. (1999) 877-881

19. Lingrand, D.: Introduction au Traitement d'Images. 2nd edn. Vuibert, Paris, France (February 2008)

20. Bres, S., Jolion, J.M., Lebourgeois, F.: Traitement et analyse des images numériques. Hermès (2003)

21. Forssén, P.E., Moe, A.: Contour descriptors for view-based object recognition. Technical Report LiTH-ISY-R-2706, Dept. EE, Linköping University, SE-581 83 Linköping, Sweden (September 2005)

22. Sun, J., Wu, X.: Shape retrieval based on the relativity of chain codes. In: MCAM07. (2007) 76-84

23. Sajjanhar, A., Lu, G.: A grid-based shape indexing and retrieval method. Australian Computer Journal 29(4) (1997) 131-140

24. Shahabi, C., Safar, M.: An experimental study of alternative shape-based image retrieval techniques. Multimedia Tools Appl. 32(1) (2007) 29-48

25. Prokop, R., Reeves, A.: A survey of moment-based techniques for unoccluded object representation and recognition. GMIP 54(5) (September 1992) 438-460

26. Chen, C.: Improved moment invariants for shape discrimination. Pattern Recognition 26(5) (May 1993) 683-686

27. Mercimek, M., Gulez, K., Velimumcu, T.: Real object recognition using moment invariants. Sadhana 30(6) (2005) 765-775 


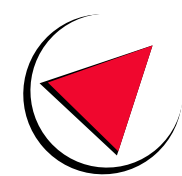

$\mathbf{L} \quad \mathbf{F} \mathbf{C}$

Laboratoire d'Informatique de l'université de Franche-Comté UFR Sciences et Techniques, 16, route de Gray - 25030 Besançon Cedex (France)

LIFC - Antenne de Belfort : IUT Belfort-Montbéliard, rue Engel Gros, BP 527 - 90016 Belfort Cedex (France) LIFC - Antenne de Montbéliard : UFR STGI, Pôle universitaire du Pays de Montbéliard - 25200 Montbéliard Cedex (France) 